Check for updates

Cite this: RSC Adv., 2019, 9, 28548

\title{
Doxorubicin loaded ferritin nanoparticles for ferroptosis enhanced targeted killing of cancer cells
}

\author{
Runlin Yang, (D) † Yaoqi Li, $\uparrow$ Xinyu Wang, (D)* Junjie Yan, (D) Donghui Pan, Yuping Xu, \\ Lizhen Wang and Min Yang (iD *
}

In recent years, ferroptosis has been investigated widely as a new form of cell death. Development of nanodrugs for ferroptosis induction in cancer cells may be a promising approach for cancer treatment. Here, we developed a type of nanoparticle consisting of the antitumor drug doxorubicin and exogenous ferritin. The drug loading process did not change the size of ferritin obviously. And this nanoparticle could induce the accumulation of ROS and cell ferroptosis for transferrin receptor overexpressed tumor cell, HT29. The ferroptosis process was also confirmed using inhibitors for ferroptosis. The cytotoxicity of this nanoparticle is similar to that of free DOX. This study provides a new strategy for targeting and killing transferrin receptor overexpressed tumor cells.

Received 14th June 2019

Accepted 2nd September 2019

DOI: $10.1039 / \mathrm{c} 9 \mathrm{ra} 04478 \mathrm{~g}$

rsc.li/rsc-advances

two mechanisms. ${ }^{11}$ One is inhibiting DNA replication and RNA synthesis in cells by inserting into the base pairs of the DNA strands. ${ }^{12}$ Another is that DOX can act as an electron acceptor in redox reactions and then be oxidized to semiquinone free radical, which causing oxidative damage to cellular membranes, proteins and DNA by producing ROS. ${ }^{12-14}$ However, the cytotoxicity of DOX result in some side effects, especially in myocardial lesions and cardiac failure. ${ }^{15-17}$ To reduce the side effects of DOX in vivo, various formulations of DOX have been investigated in clinical or preclinical fields including encapsulated in nanomaterial, such as liposome, protein and PLGA nanoparticles. ${ }^{18-20}$

Nanomaterials have shown to great potential in encapsulating and transporting drugs, penetrating cell membranes and releasing drugs in tumor cells through the enhanced permeability and retention (EPR) effect, ${ }^{21}$ which augment the accumulation of drugs in tumor cells but reduce that in normal cells. ${ }^{22}$ Ferritin (Fn), a universal intracellular protein that stores iron and releases it in a controlled fashion, was designed to encapsulate DOX for targeting delivery drugs to transferrin receptor 1 (TFR1) overexpressed tumor cells, ${ }^{23,24}$ human leukemia (HL-60), human colorectal carcinoma (Lovo), human breast adenocarcinoma (MDA-MB-468), human liver carcinoma (Hep G2), human cervical adenocarcinoma (HeLa), human breast (MCF-7), human small cell lung carcinoma (NCIH69). ${ }^{25,26}$ Ferritin, with DOX loaded, could directly interact with TFR1 (ref. 27) on the membranes of tumor cells and subsequently internalize and release DOX within the cells. By this way, DOX was released in tumor cells for therapeutic research. ${ }^{24}$

In this study, DOX was loaded into iron saturated ferritin.

Key Laboratory of Nuclear Medicine, Ministry of Health, Jiangsu Key Laboratory of Molecular Nuclear Medicine, Jiangsu Institute of Nuclear Medicine, Wuxi, 214063, China. E-mail: wangxinyu@jsinm.org; yangmin@jsinm.org

$\dagger$ These authors contributed equally to this work. also, the intracellular iron concentration can be increased by 
ferritin, which is a promoter for ferroptosis. ${ }^{28}$ As DOX can cause oxidative damage to cell in the tumor cells, it will produce ROS. ${ }^{29}$ Combined these two processes, DOX loaded ferritin may have great potential in tumor cell killing.

\section{Experimental section}

\section{Materials}

Doxorubicin hydrochloride and ferritin from equine spleen were purchased from Sigma Aldrich (Shanghai, China). Erastin and ferrostatin-1 were obtained from Selleck (Shanghai, China). Deferoxamine was obtained from Novartis (Shanghai, China), glutathione reduced form (GSH) was purchased from Tokyo Chemical Industry (Shanghai, China). $\mathrm{Fe}^{3+}\left(\mathrm{FeCl}_{3} \cdot 6 \mathrm{H}_{2} \mathrm{O}\right)$ was obtained from Sinopharm Chemical Reagent Co., Ltd, Float-ALyzer G2 Dialysis Cassettes (MWCO $3.5 \mathrm{kDa}$ ) and Tube-O-DIALYZER $^{\text {TM }}$ Micro Dialysis System (MWCO 50 kDa) were purchased from Sangon Biotech (Shanghai, China). PD-10 desalting columns were obtained from GE Healthcare. C11BODIPY was obtained from Thermo Fisher. The kits for Cell Counting Kit-8 (CCK-8), enhanced BCA protein assay, and reactive oxygen species assay, anti-actin, goat anti-rabbit IgG $(\mathrm{H}$ $+\mathrm{L})$-HRP conjugate and goat anti-mouse IgG $(\mathrm{H}+\mathrm{L})$-HRP conjugate were purchased from Beyotime Institute of Biotechnology (Jiangsu, China). Anti-GPX4 was obtained from Abcam. Anti-GPX4 and anti-actin were used as primary antibodies. Goat anti-rabbit IgG $(\mathrm{H}+\mathrm{L})$-HRP conjugate and goat anti-mouse IgG $(\mathrm{H}+\mathrm{L})$-HRP were used as secondary antibodies. All obtained chemicals were used without further purification.

\section{Synthesis of ferritin-doxorubicin (Fn-DOX)}

Ferritin was diluted into saline solution at a concentration of $5 \mathrm{mg} \mathrm{mL}{ }^{-1}$ first. Then the $\mathrm{pH}$ value of the solution was adjusted to 4 approximately by $0.05 \mathrm{~N} \mathrm{HCl}$. $2 \mathrm{~mL}$ ferritin solution was mixed with $2 \mathrm{~mL}$ DOX aqueous solution $\left(0.5 \mathrm{mg} \mathrm{mL}^{-1}\right)$ and incubated for $1 \mathrm{~h}$. After that, the products were run through a PD-10 column to remove free Dox. The $\mathrm{pH}$ value of the solution was then adjusted to 7 by $0.05 \mathrm{~N} \mathrm{NaOH}$.

The loaded Dox was quantified using a fluorescence spectrometer (LS55, PerkinElmer) by comparing to a standard curve.

\section{Characterization}

The size distribution of Fn-DOX were measured by dynamic light scattering (DLS) using a Zetasizer Nano ZSE (Malvern Instruments, Ltd.). The UV-vis absorption of the sample solutions was measured by a PerkinElmer Lambda 25 UV-vis spectrophotometer. High-performance liquid chromatography was performed with Waters 1525 equipped with 2998 photodiode array detector.

\section{Drug release}

Fn-DOX was placed into a dialysis tubes (MWCO $50 \mathrm{kDa}$ ) and dialysis in $50 \mathrm{~mL}$ PBS (pH 7.4 or 5) for $24 \mathrm{~h}$. The solutions outside the dialysis tubes were collected at various time point. The DOX concentrations were determined by HPLC analysis.

\section{Cell culture}

Human hepatoma cell line (Hep G2), and Human colorectal adenocarcinoma grade II cell line (HT29) were obtained from the Cell Bank in Shanghai Institute of Cell Biology, China. The Hep G2 cells were cultured in a humidified atmosphere of a $37{ }^{\circ} \mathrm{C}$ incubator with $5 \% \mathrm{CO}_{2}$ in DMEM medium supplemented with $10 \%$ fetal bovine serum and $0.1 \%$ penicillinstreptomycin. HT29 cells were cultured in McCoy's 5A medium supplemented with $10 \%$ fetal bovine serum and $0.1 \%$ penicillin-streptomycin.

\section{Cell uptake for Fn-DOX}

The uptake of Fn-DOX was evaluated by flow cytometric in Hep G2 and HT29 cells. Cells with media were added into 6-well plate $\left(3 \times 10^{5}\right.$ cells per well $)$ and incubated for overnight at $37{ }^{\circ} \mathrm{C}$. Cells were then treated with $0.5 \mu \mathrm{M}$ free DOX for $2 \mathrm{~h}$ or Fn-DOX, with the same concentration of free DOX $(0.5 \mu \mathrm{M})$, for $15,30,60,120 \mathrm{~min}$. All of cells were collected and evaluated by flow cytometer.

\section{Cell viability assay}

HT29 cells were seeded into 96 -well plate $\left(1 \times 10^{4}\right.$ cells per well $)$ and incubated for overnight at $37^{\circ} \mathrm{C}$. The cells were incubated with Fn-DOX, DOX, DOX-Fe (mixture of DOX and ferric chloride), and ferritin at different concentrations for $24 \mathrm{~h}$ at $37^{\circ} \mathrm{C}$, and then analyzed via the standard CCK-8 assay. To confirm FnDOX-mediated cell ferroptosis pathways, we selected several typical cell ferroptosis inhibitors for cell viability assays, including DFO, GSH and Fer-1. The cells were incubated with each inhibitor and Fn-DOX for $24 \mathrm{~h}$. The concentrations of the inhibitors were used as follows: DFO, $500 \mu \mathrm{M}$; GSH, $5 \mathrm{mM}$; Fer$1,10 \mu \mathrm{M}$. After the treatment, cell viabilities were assessed using the CCK-8 assay.

\section{Cell lipid peroxide staining}

HT29 cells were seeded with a density of $1 \times 10^{5}$ per well in 24well plates and incubated overnight. Cells were treated with agents for $24 \mathrm{~h}$. After incubation, the culture medium was replaced and cells were washed by PBS for three times. $2.5 \mathrm{~mL}$ of fresh medium containing the lipid peroxidation sensor C11BODIPY was added into each well with the final concentration of $5 \mu \mathrm{M}$ and incubating cells for another $30 \mathrm{~min}$. After washed by PBS, the cells were observed using a fluorescence microscopy (Olympus).

\section{In vitro ROS detection}

The fluorescent probe DCFH-DA was used to detect the intracellular ROS changes. HT29 cells were seeded into 6-well plate $\left(5 \times 10^{5}\right.$ cells per well) and incubated for overnight at $37{ }^{\circ} \mathrm{C}$. Then the cells were treated with DFO, GSH, Fer-1 and Fn-DOX for $24 \mathrm{~h}$. A final concentration of $10 \mu \mathrm{M}$ DCFH-DA mixed with fresh cell culture medium was added to the wells and the cells were incubated in a $37{ }^{\circ} \mathrm{C}$ incubator for $20 \mathrm{~min}$ and washed three times by PBS. Finally, cells were digested and dispersed in $500 \mu \mathrm{L}$ PBS for flow cytometry study. 


\section{Western blot analysis}

The samples were lysed. Cell lysates were prepared in Western and IP lysis buffer containing $1 \mathrm{mM}$ PMSF, $20 \mathrm{mM}$ Tris ( $\mathrm{pH}$ 7.5), $150 \mathrm{mM} \mathrm{NaCl}, 1 \%$ Triton $\mathrm{X}-100$, sodium pyrophosphate, $\beta$ glycerophosphate, EDTA, $\mathrm{Na}_{3} \mathrm{VO}_{4}$, leupeptin, and other inhibitors. The enhanced BCA protein assay kit was used to quantify protein levels. The protein samples with loading buffer were heated at $70{ }^{\circ} \mathrm{C}$ for $10 \mathrm{~min}$ and then run on $12 \%$ Bis-Tris gels (Invitrogen Novex). After transferred to a polyvinylidene fluoride (PVDF) membrane, proteins were incubated with primary antibodies as follows: anti-GPX4 (1:15 000), and anti-actin $(1: 1000)$ at $4{ }^{\circ} \mathrm{C}$ overnight. On next day, the samples were incubated with goat anti-rabbit-HRP or anti-mouse-HRP secondary antibodies. The relative quantity of proteins was analyzed by quantity one software and normalized to that of loading controls.

\section{Statistical analysis}

All data were tested at least three times independently. Each experiment in the cell death assay were performed by 3-6 replicates. All data were represented as means $\pm \mathrm{SD}(n \geq 3)$. Student's $t$-test was performed to assess the statistical significance. A $p$-value $<0.05$ was considered statistically significant.

\section{Results and discussion}

\section{Synthesis and characterization of Fn-DOX}

The fabrication process of Fn-DOX was illustrated in Fig. 1A. The hydrodynamic size of Fn-DOX was determined by dynamic light scattering (DLS). This resulted in an average hydrodynamic diameter of $12.7 \pm 4.1 \mathrm{~nm}$ (Fig. 1C), which is a little larger than that of ferritin $(10.3 \pm 3.1 \mathrm{~nm})$ (Fig. 1B). These results suggest that the Fn-DOX did not aggregate after drug loading and remained single protein particles. TEM characterization was also performed to determine the size distribution of FnDOX (Fig. 1D and E). It showed that the average size of FnDOX is around $8 \mathrm{~nm}$ with a narrow size distribution. The UVvis-NIR spectra of Fn-DOX demonstrated a characteristic absorption peak at $490 \mathrm{~nm}$, corresponding for the component of DOX in the Fn-DOX (Fig. 2A). The drug loading efficiency is $5.27 \%$ which determined by fluorescence spectrum. To simulate the extracellular and endolysosomal environments, the release of DOX from Fn-DOX was studied at $\mathrm{pH} 7.4$ and $\mathrm{pH}$ 5.0, respectively (Fig. 2B). It was found that Fn-DOX was almost stable at $\mathrm{pH} 7.4$ with only less than $10 \%$ DOX released after $24 \mathrm{~h}$ incubation. And as the $\mathrm{pH}$ decreased to $\mathrm{pH} 5$, the DOX loaded in Fn-DOX exhibits rapid dissociation release compared to $\mathrm{pH} 7.4$, the release of DOX reaches about $80 \%$ after $24 \mathrm{~h}$. This $\mathrm{pH}$ dependent drug release behavior of Fn-DOX reveal that the DOX can be released in endolysosome.

\section{Selective uptake of Fn-DOX}

Although varies of nanodrugs have been explored to kill cancer cells through ferroptosis, such as cisplatin loaded iron oxide, ${ }^{30}$ lipid hydroperoxide nanoparticles, ${ }^{31}$ and metal-organic network encapsulated with p53 plasmid, ${ }^{32}$ the targeting ability
(A)
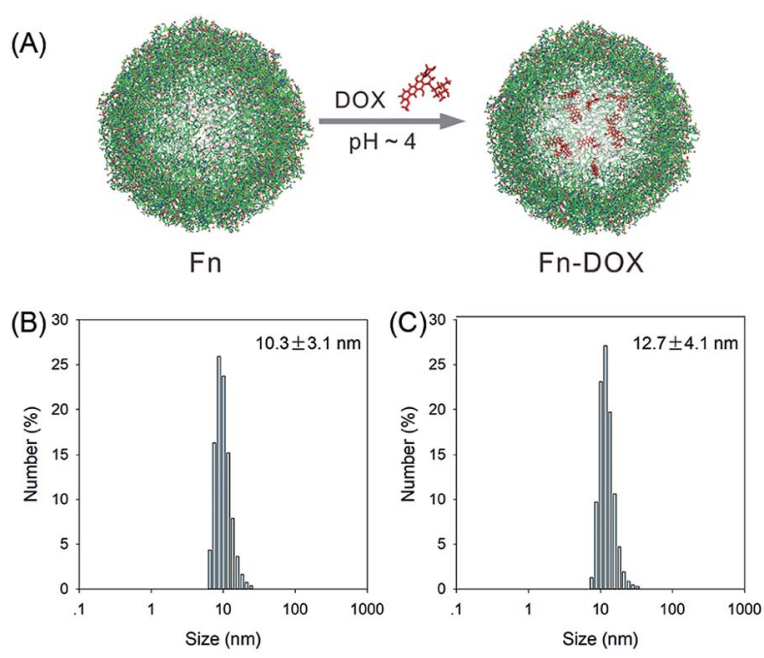

(D)
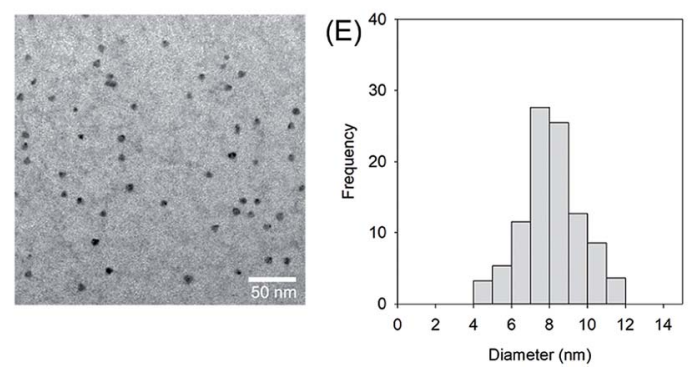

Fig. 1 (A) The schematic synthesis route of Fn-DOX nanoparticle by self-assembly. Size distribution of Fn (B) and Fn-DOX (C) determined by DLS. (D) TEM image of Fn-DOX. (E) Size distribution of Fn-DOX determined by TEM image.

was been ignored. The transferrin receptor is present on the surface of many cells and mediates iron-containing ferritin from outside the cell. In order to verify the targeting capacity of Fn-DOX on tumor cells, we selected HT29 cells (human colon adenocarcinoma cell line) with high expression of transferrin receptor and Hep G2 cells (human liver hepatocyte carcinoma cell line) with low expression of transferrin receptor. The intracellular uptake of Fn-DOX and free DOX in both cell lines were compared by flow cytometry. As shown in Fig. 3, cellular uptake of free DOX is almost identical in these two cells, this result can be verified from the mean fluorescence intensity (MFI). Whereas the uptake of Fn-DOX in these two cells showed
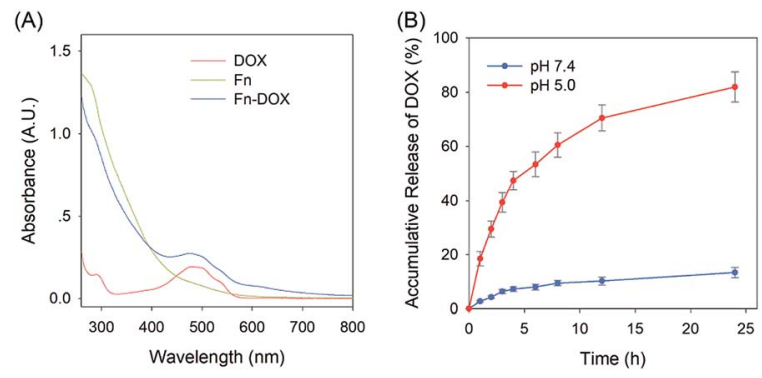

Fig. 2 (A) UV-Vis spectra of DOX, Fn and Fn-DOX in water. (B) Accumulation release of DOX from Fn-DOX under different $\mathrm{pH}$ conditions. 
(A)

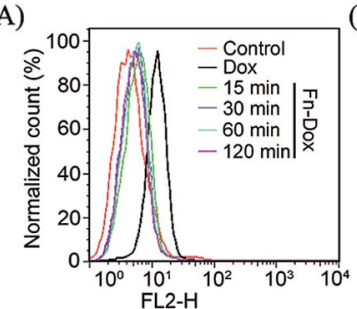

(C)

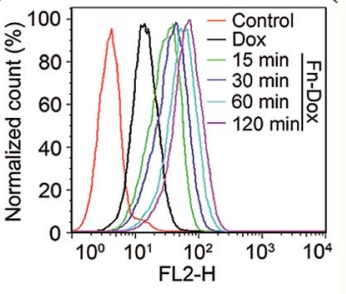

significant differences. Since HT29 cells have highly expressed transferrin receptors, more Fn-DOX can be transferred into the cells (Fig. 3C). In other words, high uptake of Fn-DOX is dependent on intracellularly high expression of transferrin receptors and exhibits time-dependent uptake. As shown in Fig. 3D, when Fn-DOX was incubated with HT29 for 120 min, the mean fluorescence intensity of the cells was approximately 16 times that of the control group. These results showed the great potential of Fn-DOX for targeting killing of cancer cells.

\section{Cytotoxicity in HT29 cells}

The cytotoxicity of Fn-DOX, DOX, DOX-Fe, and ferritin was evaluated in HT29 cells (Fig. 3A-D). Ferritin without DOX has almost no toxicity to HT29 cells within $0.5 \mathrm{mg} \mathrm{mL}^{-1}$, and the half maximal inhibitory concentration $\left(\mathrm{IC}_{50}\right)$ were $1.683,1.330$ $\mu \mathrm{M}$ for DOX, and DOX-Fe, respectively. The $\mathrm{IC}_{50}$ of Fn-DOX was $1.349 \mu \mathrm{M}$, which was closed to DOX-Fe. This may be due to the dissociation of Fn-DOX in the acidic environment in endosome and lysosome of tumor cells and then DOX released from ferritin. And the transferrin receptors targeting ability of FnDOX may also promote the cytotoxicity. These results suggest Fn-DOX got a strong cancer killing ability just like the free DOX (Fig. 4).

\section{Ferroptosis induced by Fn-DOX}

We further examined if cell death was occurring by ferroptosis pathway. Membrane lipid oxidation is one of the hallmarks of cell ferroptosis. ${ }^{\mathbf{1}, 3}$ The level of intracellular membrane lipid oxidation was measured by fluorescence microscopy. C11BODIPY as a membrane lipid oxidation detection probe, was used to incubate with the HT29 cells which was first treated by Fn-DOX, DOX, or DOX-Fe. As shown in Fig. 5, the Fn-DOX group showed highest fluorescence intensity compared with the control group, DOX, and DOX-Fe which means highest

(A)

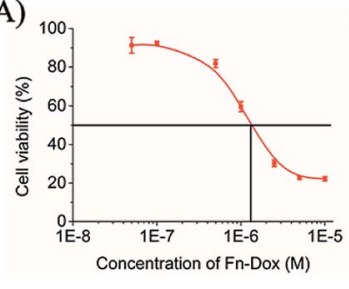

(C)
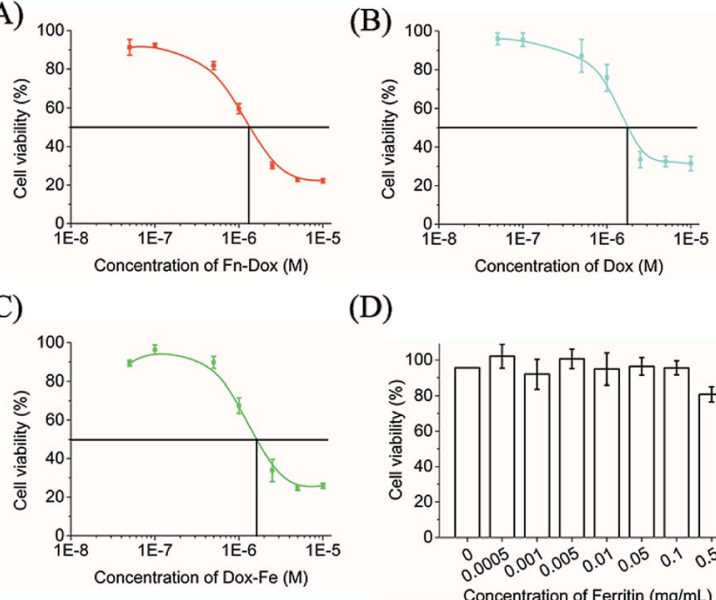

(D)

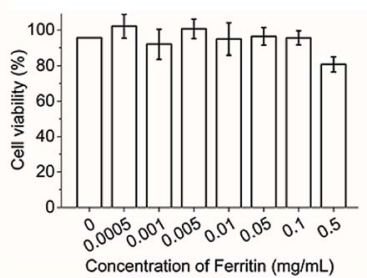

Fig. 4 In vitro cytotoxicity of Fn-DOX (A), DOX (B), DOX-Fe (C), and Fn (D) in HT29 cells for $24 \mathrm{~h}$.

membrane lipid oxidation level in cells induced by Fn-DOX. In addition, detection of changes in protein levels is also necessary to determine the form of cell death. GPX4 is a phospholipid hydroperoxidase that protects cells against membrane lipid peroxidation. ${ }^{2}$ Erastin caused GPX4 reduced synthesis in HT29 cells, suggesting that the protein reduction is involved in ferroptosis. Similarly, GPX4 expression level was reduced in FnDOX-treated cells, approximately to $70 \%$ of the control group. However, cells treated with DOX, Fe ions alone, or mixed with DOX and Fe, GPX4 levels were not significantly decreased (Fig. 6). These results indicate that ferroptosis can be induced by Fn-DOX.

\section{Inhibition of Fn-DOX induced ferroptosis}

Further studies found that cells treated with Fn-DOX while adding ferroptosis inhibitor, such as DFO, GSH and Fer-1, the increased red fluorescence of C11-BODIPY can be inhibited
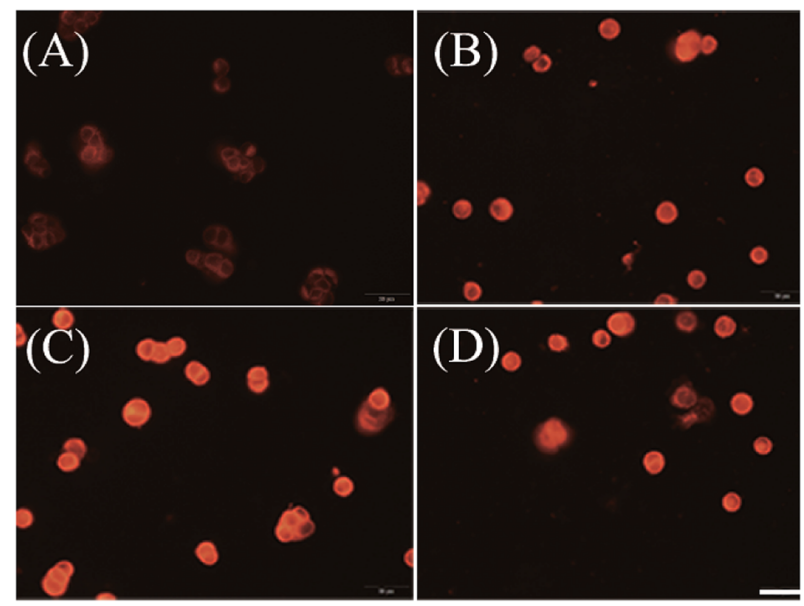

Fig. 5 Lipid peroxide in HT29 cells which were stained by C11BODIPY. (A) Control, (B) DOX, (C) Fn-DOX, and (D) DOX-Fe. The DOX concentration in (B)-(D) is $2 \mu \mathrm{M}$. Scale bar is $20 \mu \mathrm{m}$. 
(A)
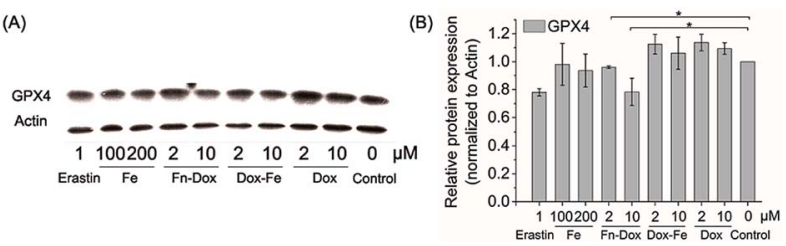

Fig. 6 (A) The expression of GPX4 in HT29 cells treated by different agents determined by western blot. (B) Quantitation of GPX4 proteins normalized to actin in by using Image $\mathrm{J}$ software. Values were presented as mean $\pm \mathrm{SD}$, each analysis was repeated three times, ${ }^{*} p<$ 0.05 .

(Fig. 7A). Next, we incubated the cells with a medium containing ferroptosis inhibitor and Fn-DOX to investigate whether the cytotoxicity of Fn-DOX was induced by ferroptosis. As shown in the Fig. 7B, DFO, GSH, and Fer-1 reduced the cytotoxicity which means part of the cytotoxicity was induced by ferroptosis process. On the other hand, a large number of reactive oxygen species (ROS) accumulate during the process of ferroptosis in cells, ${ }^{1}$ and therefore we tested it. The dichlorofluorescein diacetate (DCFH-DA) probe is a non-labeled oxidation-sensitive fluorescent probe that can be used to determine the level of intracellular ROS. ${ }^{33}$ The levels of ROS in Fn-DOX-treated cells showed a significant increase which was detected by flow cytometry (Fig. 7C). The mean fluorescence intensity of Fn-DOXtreated cells is about 65-fold higher than that of the control group. However, with the presence of DFO, GSH and Fer-1, the fluorescence intensity decreased to 50,58, and 47-fold higher than that of the control group in Fig. 5D. In general, Fn-DOX induces cell death dependent on ferroptosis which caused by
(A)

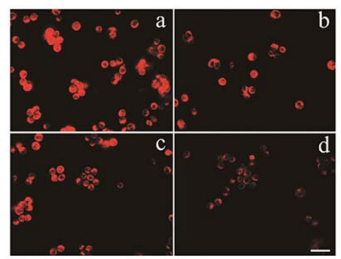

(C)

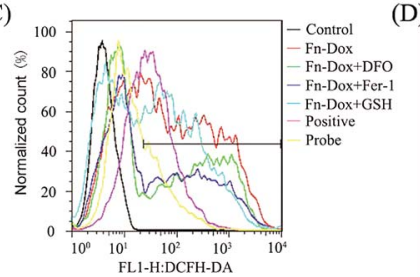

(B)

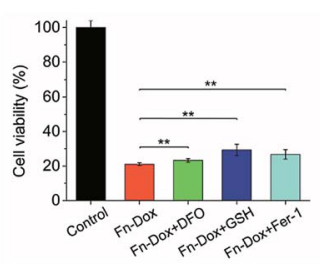

(D)

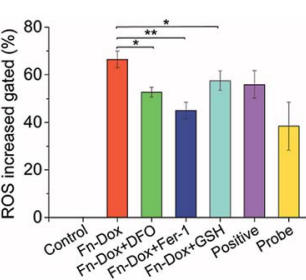

Fig. 7 (A) Lipid peroxide in HT29 cells exposed to Fn-DOX in the presence of ferroptosis inhibitors, including DFO, GSH, and Fer-1 which were stained by C11-BODIPY. (a) Fn-DOX, (b) Fn-DOX + DFO, (c) Fn-DOX + GSH, (d) Fn-DOX + Fer-1. Scale bar is $20 \mu \mathrm{m}$. (B) In vitro cytotoxicity of Fn-DOX in HT29 cells for $24 \mathrm{~h}$ in the presence of ferroptosis inhibitors, including DFO, GSH, and Fer-1. (C) ROS stained by DCFH-DA in HT29 cells treated with Fn-DOX in the presence of ferroptosis inhibitors, then were analyzed by flow cytometry. (D) Relative $\mathrm{MFI}$ of control in (A) analyzed by flow cytometry. The concentrations of DFO, GSH, Fer-1 were $500 \mu \mathrm{M}, 5 \mathrm{mM}$, and $10 \mu \mathrm{M}$. Values represented as means $\pm \mathrm{SD}(n=5)$. ${ }^{*} p<0.05,{ }^{* *} p<0.01$.

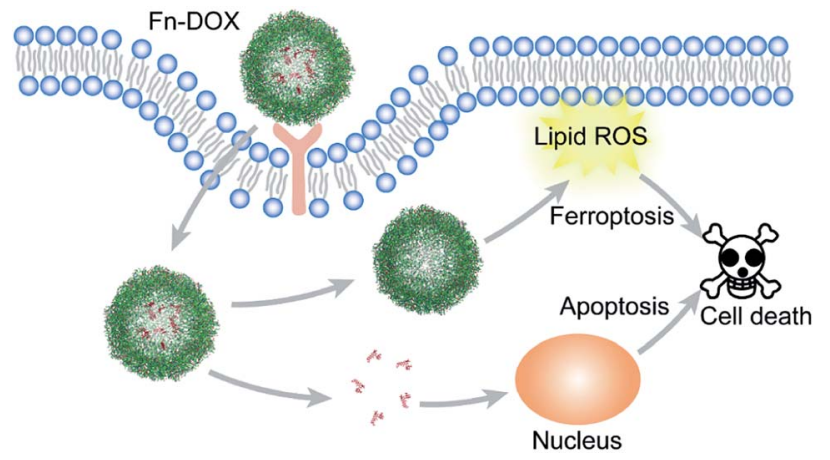

Scheme 1 Schematic illustration of cell death induction by Fn-DOX

accumulation of lipid peroxidation. These results suggest that ROS generation was also caused by iron of ferritin. And this has a critical role in Fn-DOX-induced ferroptosis. Fn-DOX induces cells to undergo a ferroptosis process that is dependent on ROS accumulation and GPX4 decline. And combined the apoptosis induced by doxorubicin, the tumor cells can be killed efficiently by Fn-DOX.

\section{Conclusions}

In summary, we designed DOX loaded iron saturated ferritin for cancer treatment (Scheme 1). The small size nanodrug with $12.7 \mathrm{~nm}$ hydrodynamic radius showed $\mathrm{pH}$ responsive drug release behavior. The HT29 cell targeting capability of Fn-DOX was confirmed by FACS measurement of the instinct fluorescence of DOX. The ferroptosis induction process by Fn-DOX was proved by fluorescent sensor and western blot. Furthermore, it was also confirmed by inhibitors of ferroptosis. And the cytotoxicity of Fn-DOX was similar to free DOX. This ferroptosis enhanced targeting killing of cancer cell by Fn-DOX provides a new insight into ferritin-based drug delivery system.

\section{Conflicts of interest}

There are no conflicts to declare.

\section{Acknowledgements}

Financial support was from the National Natural Science Foundation of China $(31671035,51803082,31971316)$, the National Significant New Drugs Creation Program (2017ZX09304021), the Jiangsu Province Foundation (BK20170204, BK20161137), the Jiangsu Provincial Medical Innovation Team (CXTDA2017024), the Jiangsu Provincial Commission of Health and Family Planning Foundation (LGY2017088, H2018069, H2017031), the Jiangsu Provincial Key Medical Discipline (ZDXKA2016017), Wuxi Science and Technology Development Foundation (WX18IIAN046), the Wuxi Commission of Health and Family Planning Foundation (Q201729) and the Innovation Capacity Development Plan of Jiangsu Province (BM2018023). 


\section{Notes and references}

1 S. J. Dixon, K. M. Lemberg, M. R. Lamprecht, R. Skouta, E. M. Zaitsev, C. E. Gleason, D. N. Patel, A. J. Bauer, A. M. Cantley and W. S. Yang, Cell, 2012, 149, 1060-1072.

2 W. S. Yang, R. Sriramaratnam, M. E. Welsch, K. Shimada, R. Skouta, V. S. Viswanathan, J. H. Cheah, P. A. Clemons, A. F. Shamji and C. B. Clish, Cell, 2014, 156, 317-331.

3 N. Yagoda, M. V. Rechenberg, E. Zaganjor, A. J. Bauer, S. Y. Wan, D. J. Fridman, A. J. Wolpaw, I. Smukste, J. M. Peltier and J. J. Boniface, Nature, 2007, 447, 864-868.

4 S. Y. Wan and B. R. Stockwell, Chem. Biol., 2008, 15, 234-245. 5 A. J. Wolpaw, K. Shimada, R. Skouta, M. E. Welsch, U. D. Akavia, D. Pe'Er, F. Shaik, J. C. Bulinski and B. R. Stockwell, Proc. Natl. Acad. Sci. U. S. A., 2011, 108, 16151-16152.

6 S. J. Dixon, Immunol. Rev., 2017, 277, 150-157.

7 Z. Cheng and Y. Li, ChemInform, 2010, 107, 748-766.

8 A. Seiler, M. Schneider, H. Förster, S. Roth, E. K. Wirth, C. Culmsee, N. Plesnila, E. Kremmer, O. Rådmark and W. Wurst, Cell Metab., 2008, 8, 237-248.

9 S. J. Dixon, D. N. Patel, M. Welsch, R. Skouta, E. D. Lee, M. Hayano, A. G. Thomas, C. E. Gleason, N. P. Tatonetti and B. S. Slusher, eLife, 2014, 3, e02523.

10 P. Mohan and N. Rapoport, Mol. Pharmaceutics, 2010, 7, 1959-1973.

11 M. Cagel, E. Grotz, E. Bernabeu, M. A. Moretton and D. A. Chiappetta, Drug discovery today, 2017, 22, 270-281.

12 C. F. Thorn, C. Oshiro, S. Marsh, T. Hernandezboussard, H. Mcleod, T. E. Klein and R. B. Altman, Pharmacogenetics Genom., 2011, 21, 440-446.

13 D. A. Gewirtz, Biochem. Pharmacol., 1999, 57, 727-741.

14 J. H. Doroshow, Proc. Natl. Acad. Sci. U. S. A., 1986, 83, 45144518.

15 M. J. Aiken, V. Suhag, C. A. Garcia, E. Acio, S. Moreau, D. A. Priebat, S. P. Chennupati and N. D. Van, Clin. Nucl. Med., 2009, 34, 762-767.

16 Y. Ichikawa, M. Ghanefar, M. Bayeva, R. Wu, A. Khechaduri, S. V. Naga Prasad, R. K. Mutharasan, T. J. Naik and H. Ardehali, J. Clin. Invest., 2014, 124, 617-630.
17 A. M. Rahman, S. W. Yusuf and M. S. Ewer, Int. J. Nanomed., 2007, 2, 567-583.

18 A. Chanan-Khan, J. Szebeni, S. Savay, L. Liebes, N. M. Rafique, C. R. Alving and F. M. Muggia, Ann. Oncol., 2003, 14, 1430-1437.

19 K. Schmidt, B. R. Gardill, A. Kern, P. Kirchweger, M. Börsch and Y. A. Muller, Proc. Natl. Acad. Sci. U. S. A., 2018, 115, 5744-5749.

20 S. Sengupta, D. Eavarone, I. Capila, G. Zhao, N. Watson, T. Kiziltepe and R. Sasisekharan, Nature, 2005, 436, 568-572.

21 D. Peer, J. M. Karp, S. Hong, R. Margalit, O. C. Farokhzad and R. Langer, Nat. Nanotechnol., 2007, 2, 751-760.

22 Y. Yuan, T. Cai, X. Xia, R. Zhang, P. Chiba and Y. Cai, Drug Delivery, 2016, 23, 3350-3357.

23 M. Liang, K. Fan, M. Zhou, D. Duan, J. Zheng, D. Yang, J. Feng and X. Yan, Proc. Natl. Acad. Sci. U. S. A., 2014, 111, 14900-14905.

24 V. Damiani, E. Falvo, G. Fracasso, L. Federici, M. Pitea, V. D. Laurenzi, G. Sala and P. Ceci, Int. J. Mol. Sci., 2017, 18, 1555.

25 T. R. Daniels, T. Delgado, G. Helguera and M. L. Penichet, Clin. Immunol., 2006, 121, 159-176.

26 T. R. Daniels, T. Delgado, J. A. Rodriguez, G. Helguera and M. L. Penichet, Clin. Immunol., 2006, 121, 144-158.

27 K. Fan, C. Cao, Y. Pan, D. Lu, D. Yang, J. Feng, L. Song, M. Liang and X. Yan, Nat. Nanotechnol., 2012, 7, 459-464.

28 G. O. Latunde-Dada, Biochim. Biophys. Acta, Gen. Subj., 2017, 1861, 1893-1900.

29 S. Y. Kim, S. J. Kim, B. J. Kim, S. Y. Rah, S. M. Chung, M. J. Im and U. H. Kim, Exp. Mol. Med., 2006, 38, 535-545.

30 P. Ma, H. Xiao, C. Yu, J. Liu, Z. Cheng, H. Song, X. Zhang, C. Li, J. Wang and Z. Gu, Nano Lett., 2017, 17, 928-937.

31 Z. Zhou, J. Song, R. Tian, Z. Yang, G. Yu, L. Lin, G. Zhang, W. Fan, F. Zhang and G. Niu, Angew. Chem., Int. Ed. Engl., 2017, 129, 6492-6496.

32 D. W. Zheng, Q. Lei, J. Y. Zhu, J. X. Fan, C. X. Li, C. Li, Z. Xu, S. X. Cheng and X. Z. Zhang, Nano Lett., 2017, 17, 284-291.

33 S. Ortelli, A. L. Costa, P. Matteucci, M. R. Miller, M. Blosi, D. Gardini, S. A. M. Tofail, L. Tran, D. Tonelli and C. A. Poland, RSC Adv., 2018, 8, 40369-40377. 